\title{
NANOFÓSSEIS CALCÁRIOS DO MIOCENO DA BACIA DO BAIXO TEJO, PORTUGAL
}

\author{
JOÃO PAULO DA SILVA LEMOS @ \& \\ GEIZE CAROLINNE CORREIA ANDRADE OLIVEIRA (D \\ Universidade Federal do Oeste do Pará, Instituto de Engenharia e Geociências, 68040-255, Santarém, PA, Brasil. \\ jao.lemoss@gmail.com,geize.oliveira@ufopa.edu.br
}

\begin{abstract}
Calcareous nannofossils of the Lower Tejo Basin Miocene, Portugal. This study presents the taxonomy, biostratigraphy, and paleoecology of calcareous nannofossils from an outcrop on the border of Foz da Fonte beach, geologically setting in the Neogene of the Lower Tejo Basin, Portugal. The taxonomic description allowed us to identify 19 calcareous nannofossils species belonging to ten genera (Helicosphaera, Pontosphaera, Reticulofenestra, Cyclicargolithus, Coccolithus, Discoaster, Sphenolithus, Umbilicosphaera, Calcidiscus, and Thoracosphaera). Based on the stratigraphic range of Discoaster druggii, Reticulofenestra lockeri, and Helicosphaera ampliaperta the section was defined in the NN2 and CN1 biozones, within the Burdigalian (lower Miocene). Quantitative analysis showed a significant variation in species diversity as a function of depth, as well as a relationship between species richness and the calcium carbonate content present in these rocks. According to these data, mainly due to abundance of Reticulofenestra spp., Coccolithus spp. and Helicosphaera spp., it is possible to infer that this was a nutrient-rich nearshore marine environment (eutrophic) influenced by warm water conditions.
\end{abstract}

Keywords: calcareous nannofossils, taxonomy, biostratigraphy, paleoecology, Neogene, Lower Tejo Basin.

RESUMO - Este estudo apresenta a taxonomia, bioestratigrafia e paleoecologia dos nanofósseis calcários presentes nas rochas que afloram às margens da praia da Foz da Fonte, geologicamente pertencentes ao Neógeno da Bacia do Baixo Tejo, Portugal. A descrição taxonômica permitiu identificar 19 espécies de nanofósseis calcários pertencentes a dez gêneros (Helicosphaera, Pontosphaera, Reticulofenestra, Cyclicargolithus, Coccolithus, Discoaster, Sphenolithus, Umbilicosphaera, Calcidiscus e Thoracosphaera). Com base na distribuição bioestratigráfica de Discoaster druggii, Reticulofenestra lockeri e Helicosphaera ampliaperta a seção foi definida pertencendo às biozonas $\mathrm{NN} 2$ e CN1, dentro do Burdigaliano (Mioceno inferior). A análise quantitativa mostrou significativa variação na diversidade de espécies em função da profundidade e, também, uma relação entre a riqueza específica e abundância com o teor de carbonato de cálcio presente nas rochas. De acordo com esses dados, principalmente pela abundância de Reticulofenestra spp., Coccolithus spp. e Helicosphaera spp. é possível inferir que tratava de um ambiente marinho próximo à costa, rico em nutrientes (eutrófico), influenciado por massas de águas quentes.

Palavras-chave: nanofósseis calcários, taxonomia, bioestratigrafia, paleoecologia, Neógeno, Bacia do Baixo Tejo.

\section{INTRODUÇÃO}

Os nanofósseis calcários e nanolitos são denominações aplicadas a partículas fósseis com dimensões inferiores 30 $\mu \mathrm{m}$. De modo geral, este grupo fóssil é dividido em duas categorias: os cocólitos e as formas associadas (nanolitos). Os cocólitos geralmente exibem forma arredonda e são provenientes da desagregação de envoltórios celulares de algas unicelulares conhecidas como cocolitoforídeos. Já os nanolitos exibem formas variadas e possuem uma origem taxonômica ainda incerta (Young \& Bown, 1997).

Esse grupo de microfósseis, de acordo com Bown \& Young (1998), possui um registro fóssil exclusivamente marinho, com hábito de vida predominantemente planctônico, composição química carbonática e ocorrência contínua desde o seu surgimento no final do Triássico até o Presente. Os nanofósseis calcários têm fornecido diversas informações que auxiliam na compreensão do passado geológico, como, por exemplo, interpretações da paleoecologia, paleotemperatura, paleoambiente, bioestratigrafia e biogeografia (Rai \& Singh, 2010).

As informações referentes às associações de nanofósseis calcários na Bacia do Baixo Tejo (BBT), Portugal, ainda são pouco documentadas na literatura. Entretanto, desde o século XVIII, com o trabalho de Almeida (1762), tornouse alvo de pesquisas geológicas e paleontológicas com o intuito de compreender os processos evolutivos que atuaram nesta bacia. Muitos destes trabalhos seguiram o ramo da paleontologia, uma vez que as unidades litoestratigráficas detêm um rico conteúdo fossilífero, como, por exemplo, o estudo de diversos grupos de microfósseis: carófitas (Marsche, 1978), ostracodes (Nascimento, 1983), foraminíferos (Legoinha, 2001), palinomorfos (Castro, 2006), dentre outros inúmeros estudos com vertebrados, invertebrados e icnofósseis que encontram-se documentados na literatura. 
Assim, o presente trabalho objetiva apresentar e discutir os resultados obtidos com a investigação de quatro amostras pertencentes ao Mioceno da Bacia do Baixo Tejo, Portugal. São apresentadas a identificação e descrição sistemática dos táxons observados, além de interpretações paleocológicas, decorrentes da quantificação das associações verificadas em cada amostra.

\section{ÁREA DE ESTUDO}

A área de estudo (Figura 1) é localmente conhecida como praia da Foz da Fonte e situa-se geograficamente a centro-oeste de Lisboa, Portugal, compreendendo parte da Península de Setúbal. O acesso até o local de estudo, partindo de Lisboa, pode ser feito através da autoestrada A5, no sentido Lisboa-Setúbal, acessando posteriormente a autoestrada do Sul, pela ponte 25 de Abril e, finalmente, através das estradas nacionais N378 e N379, respectivamente, chega-se à praia da Fonte da Foz.

\section{CONTEXTO GEOLÓGICO}

A Bacia do Baixo Tejo teve a sua origem relacionada à reativação de antigas fraturas hercinianas durante o Paleógeno, que deram origem a grábens com direção preferencial NE-W. Ela ocupa grande extensão no território português (Figura 1), estendendo-se desde o litoral de Lisboa (Península de Setúbal) até proximidades da fronteira com a Espanha (Castro, 2006).

De acordo com Pais et al. (2013), o preenchimento da bacia iniciou-se no Eoceno, com a deposição de aproximadamente $400 \mathrm{~m}$ de sedimentos grossos (rochas conglomeráticas com intercalação de arenitos) provenientes de leques aluviais, que tinham como fonte as rochas dos relevos adjacentes (Maçico Hespérico).

A passagem do Oligoceno para o Mioceno se deu de forma gradual em algumas porções da bacia, em outras foi marcada por discordância angular (Legoinha, 2001). Durante o início do Mioceno (Aquitaniano), o Oceano Atlântico invadiu a bacia e os depósitos aluviais encontram-se interdigitados por rochas de contexto marinho com abundância de macro e microfósseis.

As falésias da Foz da Fonte exibem depósitos sedimentares pertencentes ao Neógeno da Bacia do Baixo Tejo. Na porção superior, aflora um pacote sedimentar que faz parte do Mioceno, constituído por calcarenitos e margas de coloração alaranjadas e amareladas (Pais et al., 2013), onde se destaca a macrofauna de turritelas e ostras, além da microfauna composta por foraminíferos, palinomorfos, ostracodes e também os nanofósseis calcários que são discutidos nesse estudo (Manuppella et al., 1999; Castro, 2006; Legoinha, 2001).

Esta sequência superior pertencente ao Neógeno, assenta-se discordantemente sobre rochas da "Formação de Galé”, Bacia Lusitaniana, pertencentes ao Cretáceo Inferior (Albiano). Essa formação é composta por calcários argilosos amarelos, bioclásticos e margas verdes, destacando-se, dentre os fósseis, ostreídeos e orbitolinas (foraminíferos) (Cruces et al., 2002).



Figura 1. Mapa de localização da área de estudo, destacando a Bacia do Baixo Tejo (Pais et al., 2013).

Figure 1. Location map of the studied area, highlighting the Baixo Tejo Basin (Pais et al., 2013).

\section{MATERIAL E MÉTODOS}

A coleta de amostras para o desenvolvimento deste trabalho foi realizada em um afloramento às margens da Praia da Foz da Fonte, Portugal. Foram coletadas 28 amostras de forma sistemática, com espaçamento variando entre $50 \mathrm{~cm}$ a $80 \mathrm{~cm}$. Entretanto, só foi possível recuperar nanofósseis em quatro amostras, que se encontram posicionadas na coluna estratigráfica (Figura 2). O restante do material apresentou-se estéril. As rochas coletadas consistem em margas com grande abundância de macrofósseis (gastrópodes e bivalves).

As amostras foram processadas no Laboratório Multidisciplinar de Geologia I da Universidade Federal do Oeste do Pará, Santarém, PA, Brasil, onde também foi 


\section{Coluna litológica}


Figura 2. Seção estratigráfica (modificada de Antunes et al., 1999), destacando os pontos de coleta de amostra em cada camada (XA, XB, XC, XD).

Figure 2. Stratigraphic section (modified from Antunes et al., 1999) with the sampling position (XA, XB, XC, XD).

realizada a confecção de lâminas, o ensaio de calcimetria e a análise das lâminas.

A confecção das lâminas seguiu a metodologia proposta por Antunes (1997) e estas foram observadas com auxílio de um microscópio petrográfico Leica $750 \mathrm{M}$, equipado com objetivas de 100x de aumento e câmera acoplada, fazendo-se uso do software LEICA LAS EZ versão 3.4 para a tomada de fotografias de exemplares de nanofósseis selecionados.

A observação das lâminas permitiu identificar sistematicamente as espécies encontradas. Após a identificação das espécies, foi elaborada uma tabela bioestratigráfica ilustrando suas ocorrências. Para a identificação das biozonas, os zoneamentos de Martini (1971) e Okada \& Burky (1980) foram tomados como referência.
A fim de obter informações paleoecológicas, foram observadas quatro lâminas e realizada a contagem de 300 exemplares em cada uma delas, através da observação de 50 campos de visão. No entanto, algumas lâminas eram muito pobres e não foi possível chegar a 300 exemplares. Nestas foi realizada a varredura completa em toda a lâmina. Essa análise quantitativa teve como finalidade obter dados referentes a abundância (número total de exemplares contabilizados em 50 campos de visão) e a riqueza específica (número absoluto de espécies em 10 campos de visão) das espécies de nanofósseis calcários, a fim de obter informações paleoecológicas. As classes adotadas para cada uma dessas grandezas encontramse na Figura 3. 


\begin{tabular}{|c|c|c|c|c|c|}
\hline \multicolumn{2}{|c|}{ ABUNDÂNCIA } & \multicolumn{2}{c|}{$\begin{array}{c}\text { RIQUEZA } \\
\text { ESPECÍFICA }\end{array}$} & \multicolumn{2}{c}{ PRESERVAÇÃO } \\
\hline Pobre & $0-50$ & Alta & $>16$ & R & Ruim \\
\hline Comum & $51-100$ & Média & $8-16$ & M & Moderada \\
\hline Abundante & $>101$ & Baixa & $0-8$ & B & Boa \\
\hline
\end{tabular}

Figura 3. Definição das classes utilizadas para abundância, riqueza específica e preservação das espécies de nanofósseis calcários.

Figure 3. Defining the classes used for abundance, specific wealth, and preservation of the calcareous nannofossils.

De outro modo, a análise qualitativa permitiu classificar a preservação da associação de nanofósseis como um todo, sendo classificada segundo os seguintes critérios: (i) amostras com boa preservação, espécimes pouco fragmentados com nenhum sinal de dissolução; (ii) amostra com preservação moderada, a maioria dos espécimes encontravam fragmentados ou com sinais de dissolução; (iii) amostra com preservação ruim, espécimes muito fragmentados com sinais de dissolução e intercrescimento. Ainda para a análise quantitativa, foi aplicado o método matemático de Shannon \& Weaver (1949) com a finalidade de verificar de que forma a diversidade das espécies estava variando em cada profundidade e a sua relação com a riqueza específica. Esse método possui relação direta com a diversidade das espécies, onde quanto maior foi o seu valor, maior será a diversidade da associação. $O$ índice de Shannon $\left(H^{\prime}\right)$ é calculado pela seguinte equação:

$$
\mathrm{H}^{\prime}=-\sum \text { pi ln pi }
$$

onde pi é a proporção de indivíduos da espécie $i$.

Com o intuito de medir o teor de carbonato de cálcio $\left(\mathrm{CaCO}_{3}\right)$ em cada amostra e relacionar com a abundância dos táxons de nanofósseis calcários presentes em cada uma delas, foi realizado o ensaio de calcimetria com auxílio de um calcímetro modelo Bernard. Os parâmetros utilizados para a determinação do teor de $\mathrm{CaCO}_{3}$ nas rochas seguiram a metodologia desenvolvida por Melchiades \& Boschi (2006). Assim, o ensaio consistiu em deslocar uma coluna de água impulsionada pelo gás carbônico liberado pela reação química do carbonato de cálcio puro com o ácido clorídrico $(\mathrm{HCl})$ diluído. $\mathrm{O}$ volume de $\mathrm{CO}_{2}$ deslocado na bureta foi convertido por estequiometria em teor de carbonato de cálcio presente na amostra. Esse processo foi repetido, aquecendo a solução a $60^{\circ} \mathrm{C}$ para identificar a presença de dolomita.

\section{RESULTADOS}

\section{Taxonomia}

A observação das características morfológicas e ornamentações dos táxons de nanofósseis calcários permitiu agrupá-los de forma sistemática, tendo como base a proposta taxonômica de Young \& Bown (1997). Não obstante, os trabalhos de Perch-Nilsen (1985), Bown (1998), Young (1998), Aubry (1984) e o sítio Nannotax (Young et al., 2018), também auxiliaram na caracterização das espécies. Foram identificadas e descritas 14 espécies de cocolitoforídeos, quatro de nanolitos e uma espécie de dinoflagelado calcário. Todas as espécies observadas, de modo geral, exibiram boa preservação, as quais encontram-se descritas abaixo e estão representadas na Figura 4.

Reino PROTOCTISTA Margulis \& Schwartz, 2001 Filo HAPTOPHYTA Hibberd ex Ed-vardsen \& Eikrem em Edvardsen et al., 2000

Classe COCCOLITHOPHYCEAE Rothmaler, 1951 Subclasse PRYMNESIOPHYCIDAE Cavalier-Smith, 1986

Ordem ZYGODISCALES Young \& Bown, 1997

Família HELICOSPHAERACEAE Black, 1971

Helicosphaera Kamptner, 1954

Helicosphaera ampliaperta Bramlette \& Wilcoxon, 1967 (Figura 4A)

1967 Helicosphaera ampliaperta Bramlette \& Wilcoxon, p. 105 , est. 6 , figs. $1-4$.

Amplitude estratigráfica. Burdigaliano ao Langhiano (Raffi et al., 2006; Young, 1998).

Helicosphaera carteri (Wallich, 1877) Kamptner, 1954

(Figura 4B)

1877 Coccosphaera carteri Wallich, p. 347-348, est. 17, figs. 3-4, 12S, 17.

1954 Helicospahera carteri (Wallich) Kamptner, p. 21, 73, figs. 17-19.

Amplitude estratigráfica. Chattiano ao Presente (Young 1998; Young et al., 2003).

\section{Helicosphaera mediterranea Müller, 1981}

(Figura 4C)

1981 Helicosphaera mediterranea Müller, p. 45-46, est. 1, figs. 13-14.

Amplitude estratigráfica. Rupeliano ao Tortoniano (Kaenel et al., 2017).

Família PONTOSPHAERACEAE Lemmermann, 1908

Pontosphaera Lohmann, 1902

Pontosphaera multipora (Kamptner, 1948) Roth, 1970

(Figura 4D)

1948 Discolithus multiporus Kamptner, p. 5, est. 1, figs. 9a, b. 1970 Pontosphaera rigida (Kamptner) Roth, p. 861, est. 9, fig. 6 ; est. 10 , fig. 1 . 
Amplitude estratigráfica. Eoceno ao Presente (Young et al., 2003).

Discussão. De acordo com Kamptner (1948), Pontosphaera multipora exibe borda estreita e a região central possui mais de 40 poros dispostos em três anéis de forma concêntrica. Já Roth (1970) informa que os cocólitos possuem entre 50 e 60 poros com forma arredondada, que se encontram dispostos em elipses ao redor do escudo proximal. Observou-se, neste trabalho, uma variação na quantidade de anéis (dois a três) e na quantidade de poros ( 25 a 30 ).

\section{Pontosphaera japonica Takayama, 1967}

(Figura 4E)

1967 Discolithina japonica Takayama, p. 189, est. 9-10, fig. 7.

Amplitude estratigráfica. Aquitiano ao Presente (Young et al., 2003).

Família NOELAERHABDACEAE Jerkovic, 1970 emend. Young \& Bown, 1997

Cyclicargolithus Bukry, 1971

Cyclicargolithus floridanus (Hay et al.,1967)

(Figura 4F)

1967 Coccolithus floridanus Hay et al., p. 445, est. 6, figs. 1-4.

Amplitude estratigráfica. Oligoceno ao Serravaliano (PerchNielsen, 1985; Raffi et al., 2006).

Reticulofenestra Hay, Mohler \& Wade, 1966

Reticulofenestra lockeri Müller, 1970

(Figura 4G)

1970 Reticulofenestra lockeri Müller, p. 116, est. 6, figs. 3-5; est. 7, fig. 4.

Amplitude estratigráfica. Eoceno inferior ao Burdigaliano (Bown \& Newsam, 2017).

Reticulofenestra minuta Roth, 1970

(Figura 4H)

1970 Reticulofenestra minuta Roth, p. 850, est. 5, figs. 3-4.

Amplitude estratigráfica. Ypresiano ao Plioceno (Young, 1998).

Reticulofenestra haqii Backman, 1978

(Figuras 4I, J)

1978 Reticulofenestra haqii Backman, p. 110, est. 1, figs. 1-4; est. 2, fig. 10.
Amplitude estratigráfica. Aquitiano ao Zancleano (Young, 1998).

Reticulofenestra producta (Kamptner, 1963) Varol, 1989

(Figura 4K)

1963 Ellipsoplacolithus productus Kamptner, p. 172, est. 8, figs. 42, 44.

1980 Dictyococcites productus (Kamptner) Backman, p. 49-50, est. 4, figs. 1a, 2a, 6-7.

Amplitude estratigráfica. Aquitiano ao Calabriano (Kamptner, 1963; Young et al., 2018).

Reticulofenestra spp. Hay, Mohler \& Wade, 1966

(Figura 4L)

1966 Reticulofenestra spp. Hay, Mohler \& Wade, p. 379-399, est. 1-13.

Amplitude estratigráfica. Eoceno ao Presente (Young et al., 2018).

Discussão. Aqui foram agrupados Reticulofenestra com ampla abertura na região central, medindo aproximadamente metade do tamanho do cocólito. A região externa possui birrefringência elevada e encontra-se dividida em quatro quadrantes.

Ordem COCCOSPHAERALES Haeckel, 1894

Família COCCOLITHACEAE Poche, 1913

Coccolithus Schwartz, 1894

Coccolithus pelagicus (Wallich, 1877) Schiller, 1930

(Figuras 4M, N)

1877 Coccosphaera pelagica Wallich, p. 348, est. 17, figs. 1, 2, 5, 11d, 16.

1930 Coccolithus pelagicus (Wallich) Schiller, p. 246, figs. 123, a, c, d.

Amplitude estratigráfica. Daniano ao Presente (Varol, 1998; Young et al., 2003).

Família CALCIDISCACEAE Young \& Bown (1997)

Calcidiscus Kamptner, 1950

Calcidiscus spp.

(Figura 4O)

Amplitude estratigráfica. Thanetian ao Presente (Young et al., 2018).

Discussão. Foram agrupados Calcidiscus que exibiam contorno subelíptico e a região interna mostrava-se extinta. A região distal era dividida em quatro quadrantes, dispostos de forma perpendicular que exibiam alta birrefringência. 


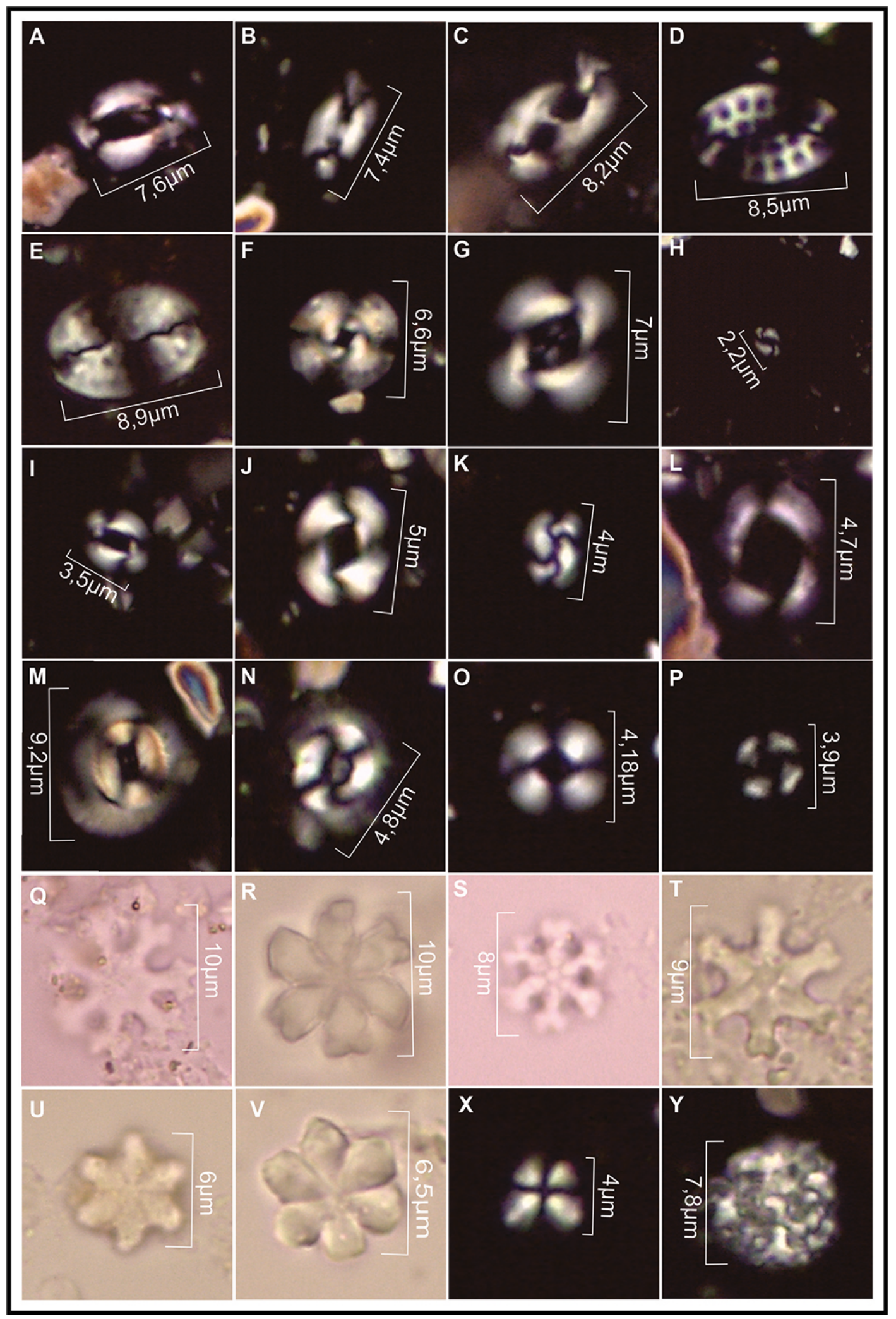

Figura 4 / Figure 4. Espécies identificadas neste estudo / Species identified in this study: A, Helicosphaera ampliaperta (0,7 m); B, Helicosphaera carteri $(0,7 \mathrm{~m}) ; \mathbf{C}$, Helicosphaera mediterranea $(15 \mathrm{~m}) ; \mathbf{D}$, Pontosphaera multipora $(0,7 \mathrm{~m}) ; \mathbf{E}$, Pontosphaera japonica $(0,7 \mathrm{~m}) ; \mathbf{F}$, Cyclicargolithus floridanus $(0,7$ $\mathrm{m})$; G, Reticulofenestra lockeri (15 m); H, Reticulofenestra minuta (1,6 m); I-J, Reticulofenestra haqii (15 m); K, Reticulofenestra producta (15 m); L, Reticulofenestra spp. (0,7 m); M-N, Coccolithus pelagicus ( $0,7 \mathrm{~m}) ; \mathbf{O}$, Calcidiscus spp. (15 m); P, Umbilicosphaera jafari $(0,7 \mathrm{~m}) ; \mathbf{Q}$, Discoaster deflandrei (3,2 m); R, Discoaster druggii (0,7 m); S-V, Discoaster spp. (0,7 m); X, Sphenolithus moriformis $(0,7 \mathrm{~m}) ; \mathbf{Y}$, Thoracosphaera spp. $(0,7 \mathrm{~m})$. 
Umbilicosphaera Lohman, 1902

Umbilicosphaera jafari Müller, 1974

(Figura 4P)

1974 Umbilicosphaera jafari Müller, p. 233, est. 1, figs 1-3; est. 4, figs. 43-44.

Amplitude estratigráfica. Aquitiano ao Ioniano (Young et al., 1998).

Ordem DISCOASTERALES Hay, 1977

Família DISCOASTERACEAE Tan, 1927

Discoaster Tan, 1927

Discoaster deflandrei Bramlette \& Riedel, 1954

(Figura 4Q)

1954 Discoaster deflandrei Bramlette \& Riedel, p. 399, est. 39, fig. 6 .

Amplitude estratigráfica. Ypresiano ao Tortoniano (Young, 1998; Kaenel et al., 2017)

Discoaster druggii Bramlette \& Wilcoxon, 1967

(Figura 4R)

1967 Discoaster druggii Bramlette \& Wilcoxon, p. 220.

Amplitude estratigráfica. Aquitiano ao Burdigaliano (Young, 1998).

Discoaster spp.

(Figuras 4S, T, U e V)

Amplitude estratigráfica. Paleoceno ao Gelasiano (Young et al., 2018).

Discussão. Os indivíduos pertencentes a esta espécie exibem tamanho e forma relativamente variada dificultando a identificação. Essa variação morfológica pode ser decorrente de processos diagenéticos como discute Aubry (1984).

Família SPHENOLITHACEAE Deflandre, 1952

Sphenolithus Deflandre, 1952

Sphenolithus moriformis (Brönnimann \& Stradner, 1960)

Bramlette \& Wilcoxon, 1967

(Figura 4X)

1960 Nannoturbella moriformis Brönnimann \& Stradner, p. 368, figs. 11-16.

1967 Sphenolithus moriformis (Brönnimann \& Stradner) Bramlette \& Wilcoxon, p. 124, est. 3, figs. 1-6.
Amplitude estratigráfica. Paleoceno ao Mioceno Superior (Perch-Nielsen, 1985; Young, 1998; Dunkley Jones et al., 2009).

Filo DINOPHYTA Raven et al., 2007

Classe DINOPHYCEAE Fritsch, 1929

Ordem THORACOSPHAERALES Tangen, 1982

Família THORACOSPHAERACEA Shiller, 1930

Thoracosphaera Kamptner, 1927

Thoracosphaera spp. Antunes, 2007

(Figura 4Y)

2007 Thoracosphaera spp. Antunes, p. 42, figs. 11-15.

Amplitude estratigráfica. Cretáceo ao Holoceno (Antunes, 2007).

Discussão. As espécies aqui agrupadas como Thoracosphaera spp. exibem semelhanças morfológicas com Thoracosphaera operculata. Entretanto, não foi possível observar o opérculo que é uma estrutura característica desta espécie e os organismos possuem dimensões diferentes.

\section{BIOESTRATIGRAFIA}

A identificação sistemática de cada táxon foi crucial para a datação bioestratigráfica. Neste estudo foi adotada a proposta de biozoneamento internacional de Martini (1971, zona NN) e Okada \& Bukry (1980, zona CN), compostos por biozonas de intervalo. Além disso, o Nannotax também foi consultado com relação à distribuição da amplitude cronoestratigráfica dos táxons reconhecidos.

Dentre os táxons identificados, observou-se que a espécie Discoaster druggii surgiu na base da biozona NN2-CN1 e foi extinta no topo na biozona NN3-CN2, e que a Reticulofenestra lockeri surgiu na NP17 e teve a sua última ocorrência no topo da NN2-CN1, como ilustra a Figura 5. O único intervalo de tempo em que essas duas espécies viveram juntas foi durante o intervalo da NN2-CN1. A biozona NN2-CN1 compreende o intervalo entre o Aquitiano e o Burdigaliano. No entanto, levando em consideração que Helicosphaera ampliaperta também surgiu na NN2, mas dentro do Burdigaliano, a associação de nanofósseis calcários estudada pertenceu ao intervalo do Burdigaliano inferior, correspondendo à biozona NN2 de Martini (1971) e à biozona CN1 de Okada \& Bukry (1980).

A idade obtida neste trabalho através de nanofósseis calcários, corresponde à idade proposta por outros autores que também realizaram estudos bioestratigráficos neste mesmo local de estudo, mas com outros grupos de microfósseis, como: a contribuição de Castro (2006) com o estudo de palinomorfos; e Legoinha (2001) com estudo de foraminíferos planctônicos e bentônicos, em especial Globigerinoides 


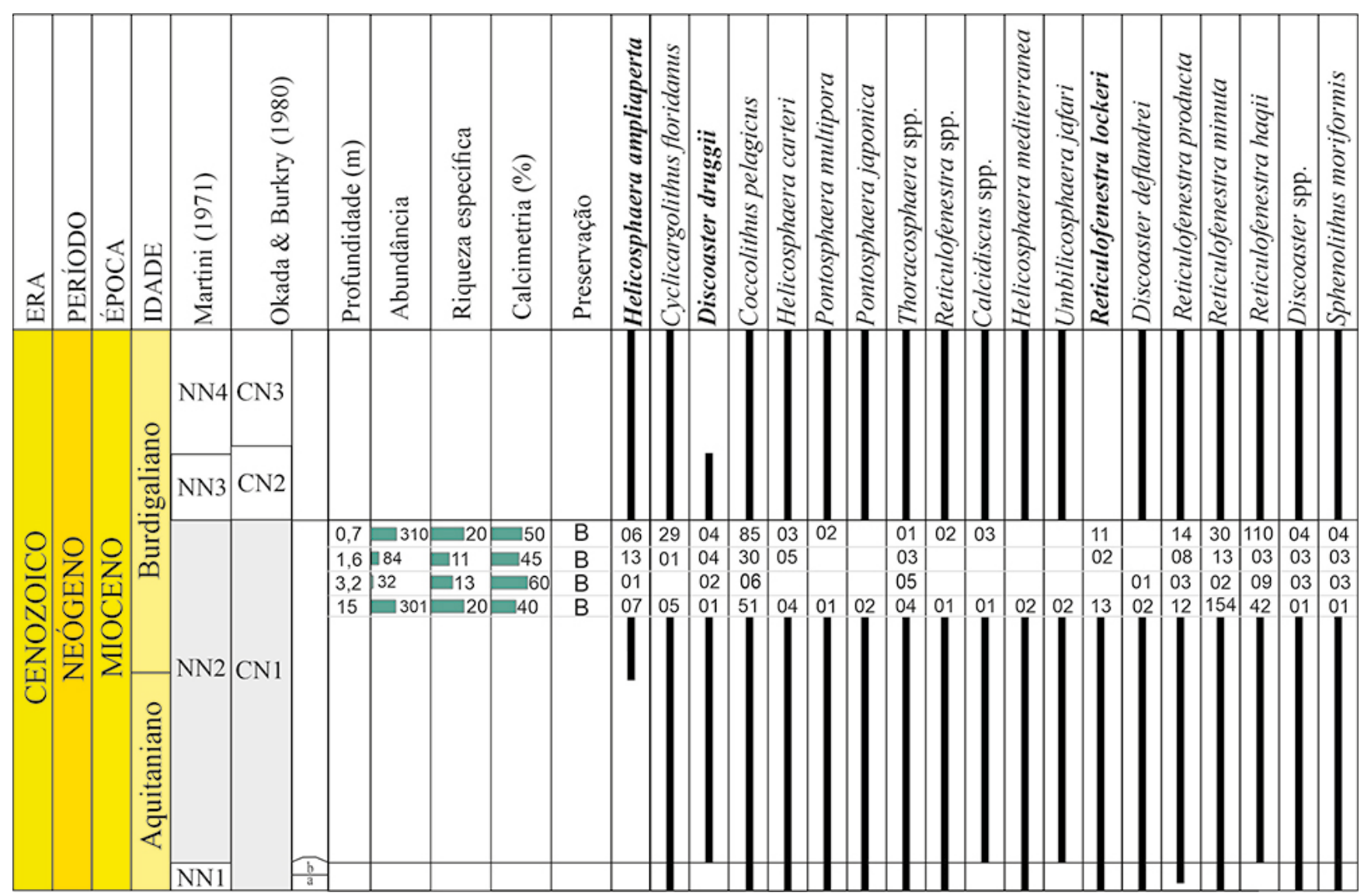

Figura 5. Zoneamento estratigráfico aplicado ao Mioceno da Bacia do Baixo Tejo, com base em nanofósseis calcários.

Figure 5. Stratigraphic zoning applied to the Lower Tejo Basin Miocene, based on calcareous nannofossils.

e a sua diversidade (G. quadrilobatus, G. trilobus, G. altiaperturus, G. immaturus, G. subquadratus, G. sacculifer e G. woodi).

\section{ANÁLISE QUANTITATIVA}

A análise quantitativa das amostras, a partir da contagem dos táxons presentes, permitiu observar que há uma variação no índice de diversidade de Shannon, riqueza específica e na abundância das espécies em relação à profundidade, como mostra a Figura 6.

Os valores obtidos para o índice de Shannon (Figura 6A) foram 1,$73 ; 2,08 ; 2,19$ e 2 , e mostram que há uma variação na diversidade das espécies de acordo com a profundidade. Isso pode ser resultado do aparecimento e desaparecimento das espécies em diferentes profundidades ou também uma resposta às condições paleocológicas daquele momento.

A diversidade das associações pode estar atrelada a vários fatores. Associações que mostram alta diversidade geralmente são características de habitats estáveis e oligotróficos, enquanto outras podem mostrar uma diversidade reduzida, típica de ambientes com altas flutuações, eutróficos e instáveis, indicando condições ecológicas extremas (Sanders, 1969; Aubry, 1992; Bollmann et al., 1993; Brand, 1994; Roth, 1994).
A

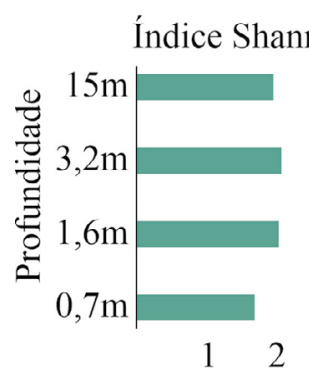

$\mathrm{C}$

Calcimetria

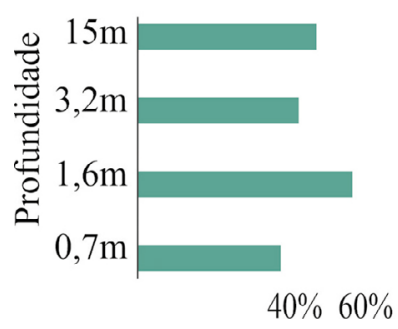

B

Riqueza Específica

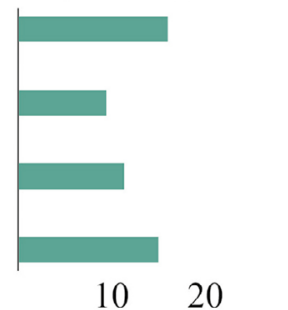

$\mathrm{D}$

\section{Abundância}



Figura 6. Gráficos destacando: A, índice de Shannon; B, riqueza específica; $\mathbf{C}$, calcimetria e $\mathbf{D}$, abundância.

Figure 6. Graphs showing: A, Shannon index; B, specific richness; C, calcimetry and $\mathbf{D}$, abundance. 
Para a riqueza específica (Figura 6B), o gráfico ilustra que houve um decréscimo na quantidade de táxons entre $0,7 \mathrm{~m}$ e $1,6 \mathrm{~m}$, seguido por uma ascensão entre $3,2 \mathrm{~m}$ e $15 \mathrm{~m}$. O gráfico da riqueza específica teve comportamento muito semelhante com o gráfico de abundância (Figura 6D), seguindo o mesmo padrão. Isso mostra que os fatores ecológicos que afetaram a quantidade de espécies encontradas (riqueza específica) em cada profundidade, afetaram também o número de indivíduos pertencentes a cada espécie (abundância). Ainda referente à abundância, as amostras das profundidades $0,7 \mathrm{~m}$ e $15 \mathrm{~m}$ foram classificadas como abundantes, enquanto as amostras $1,6 \mathrm{~m}$ e 3,2 m foram classificadas como pobres.

A tendência observada nos gráficos de abundância e riqueza específica seguiu um padrão análogo com o gráfico de calcimetria (Figura 6C) a partir de 1,6 m. Esse comportamento teve apenas uma pequena mudança na profundidade $0,7 \mathrm{~m}$. Assim, é possível observar uma relação entre esses últimos parâmetros, ou seja, o comportamento da riqueza de espécies e a abundância podem afetar o teor de carbonato de cálcio presente nas rochas. Assim, acredita-se que a calcimetria possa ser uma ferramenta para auxiliar na interpretação da variação da abundância e a riqueza específica, sendo que o teor de carbonato de cálcio presente nas amostras variou entre $40 \%$ e $60 \%$, correspondendo a rochas margosas.

\section{PALEOECOLOGIA}

Para a interpretação paleoecológica foi levado em consideração principalmente a abundância de cada táxon identificado. Assim, dentre os gêneros identificados de Reticulofenestra, neste trabalho, as espécies $R$. minuta, $R$. haqii, $R$. producta, $R$. lockeri e Reticulofenestra spp., dominam fortemente a associação de nanofósseis calcários, com $61,30 \%$ (Figura 7 ).

De acordo com Müller (1976), a grande abundância de Reticulofenestra no mar da Noruega-Groenlândia e noroeste da Europa provavelmente pode ser explicada pela água fria.
As espécies deste gênero são menos abundantes em várias partes do Atlântico Norte que foram influenciadas durante o Meso-oligoceno por correntes mais quentes, bem como pela moderna Corrente do Golfo. De acordo com Haq (1980), uma grande abundância de R. minuta foi documentada ao longo das margens continentais, tendo como preferência águas mornas. Essa abundância geralmente indica ambientes eutróficos devido ao escoamento continental, portanto, considera-se um táxon resistente e oportunista, com ampla tolerância ecológica, capaz de se propagar em condições ricas em nutrientes (Aubry, 1992; Flores et al., 1995). Wade \& Bown (2006) apontam que blooms de R. minuta estão relacionados a um alto estresse ambiental, pois esta espécie responde rapidamente a mudanças ambientais e domina condições onde outras espécies não podem competir. Gartner et al. (1983) sugerem que variações na abundância relativa de $R$. minuta se correlacionam com mudanças na dinâmica dos nutrientes e, ainda, que o tamanho diminuto dos cocólitos pode estar associado às flutuações sazonais de temperatura e nutriente.

Coccolithus spp. (Coccolithus pelagicus) é a segunda espécie mais representativa nas amostras, com $24,30 \%$ da abundância total (Figura 7). Entretanto, essa abundância exibiu uma significativa variação na quantidade de indivíduos em função da profundidade como mostra a Figura 8F. De acordo com McIntyre \& Bé (1967), C. pelagicus é abundante em sedimentos do Mioceno em oceano aberto, em especial regiões do Atlântico Norte, muito comum em águas de baixa salinidade. Rahman \& Roth (1990) informam que essa espécie teve um longo alcance, fornecendo informações paleoclimáticas do Mioceno ao Pleistoceno. Durante o início do Cenozoico, a espécie evoluiu principalmente em áreas tropicais e migrou para regiões polares durante o Cenozoico médio (Haq \& Lohman, 1976). Além do mais, mostra preferência por massas de água com baixas temperaturas (Okada \& McIntyre, 1979) e tem afinidade por águas ricas em nutrientes, sendo documentada na frente de ressurgência portuguesa, onde está especificamente associada à zona de ressurgência de intensidade moderada (Cachão \& Moita, 2000).

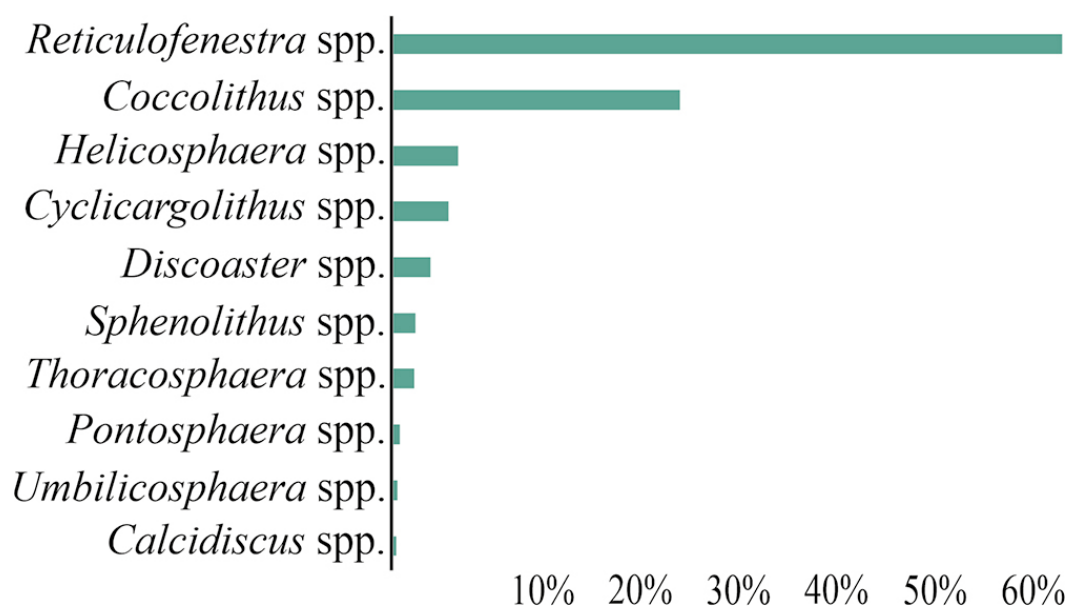

Figura 7. Abundância dos gêneros de nanofósseis calcários.

Figure 7. Calcareous nannofossil genus abundance. 
Helicosphaera spp. (H. ampliaperta, H. carteri, $H$. mediterranea) exibiu abundância de 5,6\% (Figura 7), sendo o terceiro gênero mais abundante. Helicosphaera ampliaperta (Figura 8G) foi a espécie mais abundante desse grupo e esteve presente em todas as amostras, seguida por $H$. carteri (Figura $8 \mathrm{H}$ ) que apareceu em três amostras e H. mediterranea (Figura 8I) visualizada em apenas uma amostra. As espécies de Helicosphaera ocorrem comumente em sedimentos hemipelágicos, geralmente estão ausentes em sedimentos pelágicos e são capazes de prosperar em ambientes rasos e eutróficos (Perch-Nilsen, 1985). Além disso, são comuns em zonas de ressurgência, com alto aporte de nutrientes (Schmidt, 1978; Giraudeau, 1992). Edwards (1968) acrescenta que a atual $H$. carteri é mais abundante em ambientes marinhos tropicais quentes, no entanto, também está presente em ambientes temperados e frios. Já $H$. ampliaperta mostra preferência por águas frias (Martini \& Worsley, 1971).

Os demais grupos de espécies possuem abundância inferior a 5\%, como Cyclicargolithus spp. (Cyclicargolithus floridanus) com 4,8\% (Figura 7), que foi a quarta espécie mais abundante, encontrada em apenas três amostras, mostrando grande oscilação na quantidade de indivíduos em cada amostra (Figura 8J). De acordo com Melinte (2005), C. floridanus tem preferência por águas quentes e ricas em nutrientes. Além disso, Haq (1980) informa que essa espécie era claramente cosmopolita durante o Mioceno, ocorrendo tanto no Hemisfério Norte quanto no Sul.

A abundância de Discoaster spp. (D. deflandrei, D. druggii e Discoaster spp.) foi de 3,3\% (Figura 7). Discoaster druggii foi a espécie mais abundante (Figura 8K), aparecendo em todas as amostras, seguido por Discoaster spp. (Figura 8L), que também foi observada em todas as amostras, e D. deflandrei (Figura $8 \mathrm{M})$ observada apenas em duas amostras. O gênero Discoaster é muito diversificado e bastante comum em ambientes oligotróficos, com massas de água quentes, profundas e estáveis (Haq, 1980; Lohmann \& Carlson, 1981; Flores \& Sierro, 1987; Chepstow-Lusty et al., 1989, 1992; Aubry 1992; Young, 1998). Apesar da grande maioria das espécies mostrarem afinidade ecológica por águas tropicais e subtropicais, D. deflandrei é tolerantes ou prefere águas mais frias (Aubry, 1984; Rahman \& Roth, 1990; Chira \& Mãrunteanu, 1999).

O grupo Sphenolithus spp. (Sphenolithus moriformis) exibiu $2 \%$ da abundância total das espécies. Esteve presente em todas as amostras analisadas (Figura $8 \mathrm{~N}$ ), mesmo com abundância muito baixa. Acredita-se que Sphenolithus spp. compartilhe o mesmo nicho ecológico que Discoaster spp. Assim, são comuns em ambientes de água morna, oligotrófica e estáveis (Haq \& Lohmann, 1976; Haq, 1980; Lohmann \& Carlson, 1981; Perch-Nielsen, 1985; Aubry, 1992).

Thoracosphaera spp. exibiu $1,9 \%$ da abundância total (Figura 7). Foi registrada em toda a seção estudada, com uma abundância muito baixa (Figura 80). Segundo Höll et al. (1998), Thoracosphaera prospera em águas com baixo teor de nutrientes, sendo que algumas espécies mostram preferência por águas quentes. Entretanto, as preferências ecológicas que cada espécie possui são muito específicas e não é possível usar todo o grupo como um indicador de águas oligotróficas ou quentes.
Pontosphaera spp. mostrou $0,7 \%$ da abundância total, sendo que $P$. multipora esteve presente em duas amostras, enquanto $P$. japonica em uma única amostra (Figuras $8 \mathrm{P}-\mathrm{Q}$ ). As espécies deste grupo são consideradas eutróficas (Aubry, 1992) e abundantes em condições marinhas estáveis, tolerando apenas pequenas flutuações de salinidade. Pontosphaera multipora prefere ambiente de plataforma rasa, sendo incomum em ambientes de oceano aberto (Perch-Nielsen, 1985).

O gênero Umbilicosphaera teve abundância igual a 0,5\% do total. Umbilicosphaera jafari esteve presente em duas amostras (Figura 8R). As afinidades paleoecológicas dessa espécie ainda são bastante discutidas. De acordo com Wade \& Bown (2006), U. jafari teve uma ampla distribuição durante o Neógeno, com alta abundância em ambientes marinhos restritos ou próximos à costa. Também pode estar relacionada a águas quentes, com pouca concentração de nutrientes (Auer et al., 2014).

Com base na análise quantitativa de nanofósseis na seção estudada, as espécies Reticulofenestra minuta, juntamente com Coccolithus pelagicus e Helicosphaera spp., dominam a associação. A espécie $R$. minuta, como discutido anteriormente, tem preferência por águas mais quentes com disponibilidade de nutrientes, enquanto $C$. pelagicus tem preferência por águas frias e por ambientes eutróficos. As espécies do grupo Helicosphaera spp. foram registradas tanto em ambientes com águas quentes, quanto de águas frias. Levando em consideração a dominância de $R$. minuta $(61,3 \%)$, acredita-se que o ambiente naquele momento poderia apresentar temperaturas mais quentes. A dominância desses três táxons, os quais têm em comum a afinidade por regiões próximas à costa, com ampla disponibilidade de nutriente, pode estar relacionada com a proximidade do continente, indicando um aporte continental, ou mesmo estar associada à zona de ressurgência que ocorre na costa portuguesa (Cachão $\&$ Moita, 2000).

Apesar da considerável diversidade de táxons, as outras espécies encontradas neste trabalho mostraram uma abundância muito baixa (inferior a 5\%). Algumas espécies compartilham semelhanças com Coccolithus pelagicus, Reticulofenestra minuta e Helicosphaera spp., que foram as espécies que dominaram a associação, como é o caso de Pontosphaera spp. e Cyclicargolithus floridanus, as quais têm preferência por zonas eutróficas. $\mathrm{O}$ outro grupo de espécies identificado mostra afinidades ecológicas opostas em relação às espécies dominantes, como, por exemplo, Sphenolithus spp., Discoaster spp. (exceto D. deflandrei), Umbilicosphaera spp. e Thoracosphaera spp., que, de modo geral, estão relacionadas com o baixo aporte de nutriente. Assim, os resultados das análises quantitativas, baseando-se na abundância relativa dos táxons de nanofósseis, indicam um ambiente marinho costeiro, com alto aporte de nutrientes. Entretanto, acredita-se que esse ambiente seja instável e flutuante, pela ocorrência comum de táxons que refletem preferências ecológicas opostas e pela abundância de $R$. minuta, que segundo Wade \& Bown (2006) indicam essas condições de alto stress ambiental. 


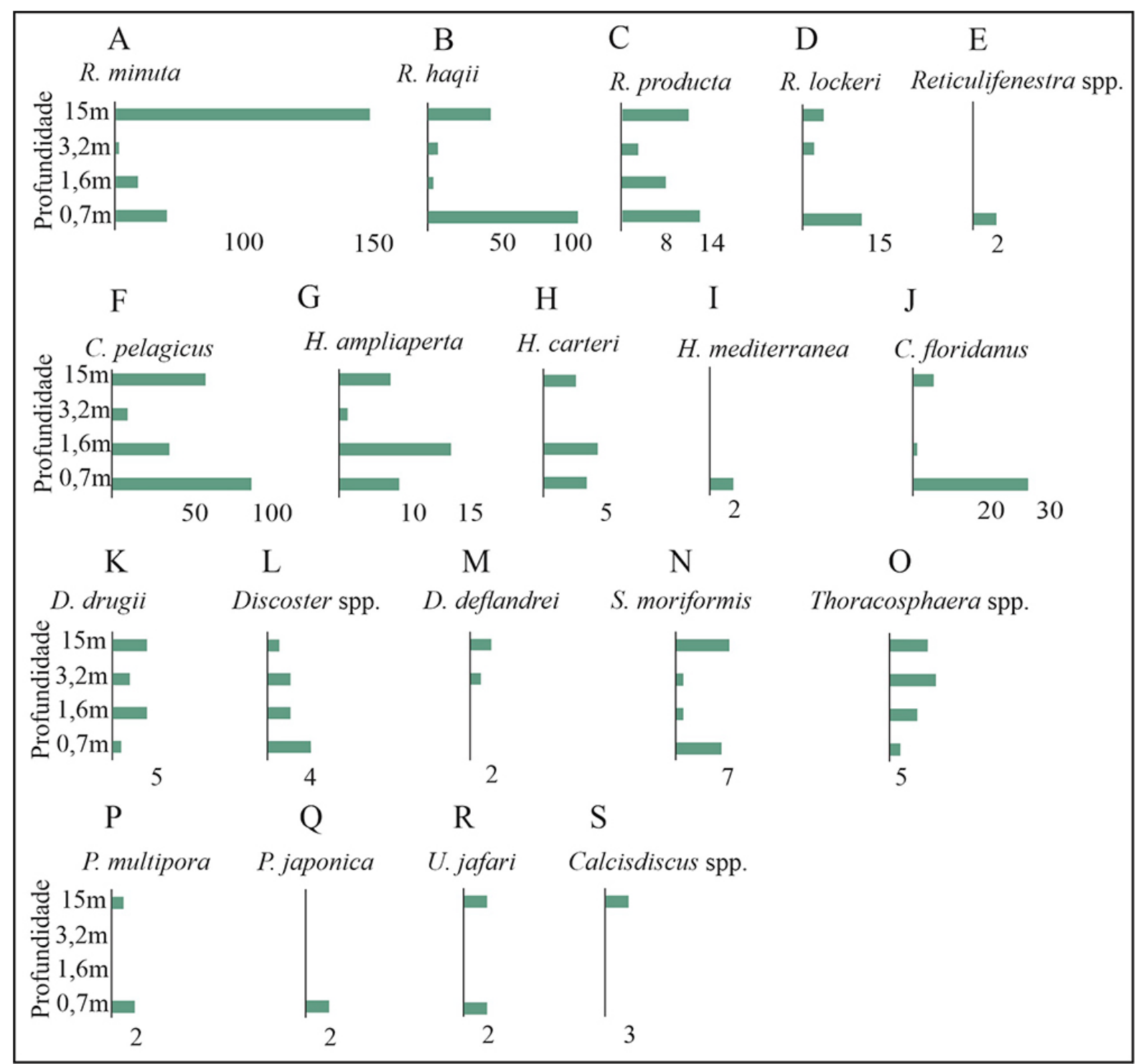

Figura 8. Abundância de cada espécie identificada em função da profundidade.

Figure 8. Identified species abundance as a function of depth.

Além da Bacia do Baixo Tejo, outras regiões sob influência do Oceano Atlântico Norte registraram a ocorrência de uma associação de nanofósseis calcários muito similar a que encontra-se descrita neste estudo (Figura 9), o que pode refletir afinidades paleoecológicas semelhantes, como, por exemplo, as bacias Canárias, nordeste do Atlântico Norte; Planície Abissal da Madeira; Sudeste da Espanha; Planície Abissal Ibéria; Sul da Islândia; Oeste da África; e Carolina do Norte (Steinmetz, 1979; Muza et al., 1987; Kaenel \& Villa, 1996; Howe \& Sblendorio-Levy, 1998; Sblendorio-Levy \& Howe, 1998; Lancis et al., 2010; Albasrawi, 2016).

\section{CONCLUSÕES}

O presente estudo corrobora dados referentes ao Neógeno da Bacia do Baixo Tejo, Portugal. O desenvolvimento da pesquisa possibilitou identificar e descrever sistematicamente
19 espécies de nanofósseis calcários pertencentes a 10 gêneros diferentes. Após a identificação das espécies, o intervalo de ocorrência de Discoaster druggi juntamente com Reticulofenestra lockeri e Helicosphaera ampliaperta permitiu concluir que as rochas estudadas fazem parte do Burdigaliano médio, dentro das biozonas CN1 de Okada \& Bukry (1980) e NN2 de Martini (1971), e correspondem com as idades obtidas por outros estudos a partir de foraminíferos e palinomorfos presentes nas rochas que afloram na Praia da Foz da Fonte.

A análise quantitativa da associação de nanofósseis analisada possibilitou concluir que existe uma significativa diversidade de espécies evidenciada pelo índice de Shannon, sendo que a abundância e a riqueza específica seguem uma tendência muito semelhante em função da profundidade. Inicialmente, tanto a riqueza específica quanto a abundância sofrem um decréscimo, seguido por uma ascensão, mostrando que pode ter sido afetada pelos mesmos fatores paleoecológicos 


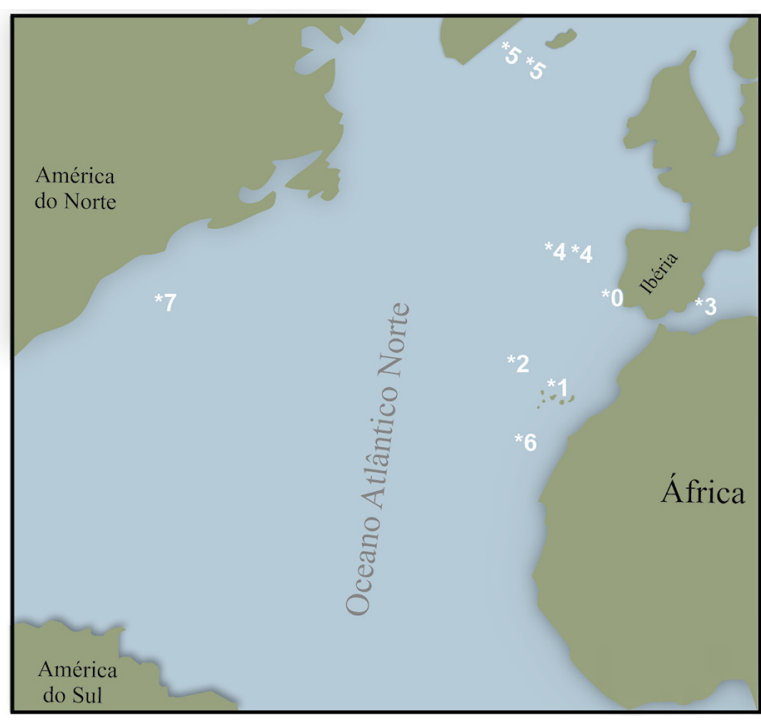

Figura 9. Mapa paleogeográfico do Mioceno (modificado de Scotese, 2014) correlacionando o local de estudo com outras regiões sob influência do Atlântico Norte: *0, local de estudo; *1, bacias Canárias (Howe \& SlendorioLevy, 1998); *2, Planíce Abissal da Madeira (Sblendorio-Levy \& Howe, 1998); *3, Sudeste da Espanha (Lancis et al., 2010); *4, Planíce Abissal da Ibéria (Kaenel \& Villa, 1996); *5, Sul da Islândia (Steinmetz, 1979); *6, Oeste da África (Albasrawi, 2016); *7, Carolina do Norte (Muza et al., 1987).]

Figure 9. Paleogeographic map of the Miocene (modified from Scotese, 2014) correlating the study site with other regions under the influence of the North Atlantic: *0, studied site; *1, Canary basins (Howe \& Sblendorio-Levy, 1998); *2, Abyssal Plain of Madeira (Sblendorio-Levy \& Howe, 1998); *3, Southeastern Spain (Lancis et al., 2010); *4, Abyssal Plain of Iberia (Kaenel \& Villa, 1996); *5, Southern Iceland (Steinmetz, 1979); *6, West Africa (Albasrawi, 2016); *7, North Carolina (Muza et al., 1987).

(temperatura, profundidade, disponibilidade de nutrientes). A calcimetria também acompanha essa tendência, e mostra que uma mudança na porcentagem de carbonato de cálcio presente em cada amostra pode estar relacionada com a variação na riqueza específica e abundância.

Além disso, a abundância de espécies dos gêneros Reticulonestra, Coccolithus e Helicosphaera possibilitou inferir que os estratos rochosos estudados foram depositados em um ambiente marinho costeiro, possivelmente com grande aporte de nutrientes, que podem estar relacionados com o aporte continental ou provenientes da zona de ressurgência que ocorre na frente portuguesa. Com base na dominância de Reticulofenestra spp., acredita-se que as massas de águas eram relativamente mornas. Tais características são correlatas com outras regiões sob influência do Oceano Atlântico Norte, que exibem um conteúdo de microfósseis idêntico.

\section{AGRADECIMENTOS}

Os autores agradecem à Universidade Federal do Oeste do Pará pelo financiamento desta pesquisa através do Edital 10/2018 PROPPIT-UFOPA, ao R.S. de Oliveira (UFOPA) pela revisão crítica do texto, ao J.C. Kullberg (Universidade Nova de Lisboa) pelo auxílio no trabalho de campo e aos dois revisores anônimos por sua cuidadosa revisão e sugestões que nos ajudaram a perceber pontos importantes que certamente melhoraram a versão final deste manuscrito.

\section{REFERÊNCIAS}

Albasrawi, W.A. 2016. Early Miocene quantitative calcareous nannofossil biostratigraphy from the tropical Atlantic. University of Nebraska-Lincoln, Dissertação de Mestrado, 56 p.

Almeida, T. 1762. Recreasaõ Filozofica, ou Dialogo Sobre a Filozofia Natural para intrusã̃ de pesoas curiozas, que nã̃ frequentáraõ as aulas. Tomo VI e ultimo. Trata dos Ceos e do Mundo. Lisboa, Oficina de Miguel Rodrigues, p. 400-418.

Antunes, M.T.; Elderfield, H.; Legoinha, P.A. \& Pais, J. 1999. A stratigraphic framework for the Miocene from the Lower Tagus Basin (Lisbon, Setúbal Península, Portugal). Depositional sequences, biostratigraphy and isotopic ages. Revista de la Sociedad Geológica de España, 12:3-15.

Antunes, R.L. 1997. Introdução ao estudo dos nanofósseis calcários. Rio de Janeiro, Universidade Federal do Rio de Janeiro, 115 p.

Antunes, R.L. 2007. Nanofósseis calcários do Quaternário da margem continental brasileira. Rio de Janeiro, CENPES/ PETROBRAS, Série Ciência Técnica Petróleo, 76 p.

Aubry, M.P. 1984. Handbook of Cenozoic calcareous nannoplankton. Book 1: Ortholithae (Discoasters). New York, American Museum of Natural History, Micropaleontology Press, 266 p.

Aubry, M.P. 1992. Late Paleogene calcareous nannoplankton evolution: a tale of climatic deterioration. In: D.R. Prothero \& W.A. Berggren (eds.) Eocene-Oligocene Climatic and Biotic Evolution, Princeton University Press, p. 272-309.

Auer, G.; Piller, W.E. \& Haarzhauser, M. 2014. High-resolution calcareous nannoplankton palaeoecology as a proxy for small-scale environmental changes in the Early Miocene. Marine Micropaleontology, 111:53-65. doi:10.1016/j. marmicro.2014.06.005

Backman, J. 1978. Late Miocene-Early Pliocene nannofossil biochronology and biogeography in the Vera Basin, SE Spain. Stockholm Contributions in Geology, 32:93-114.

Backman, J. 1980. Miocene-Pliocene nannofossils and sedimentation rates in the Hatton-Rockall Basin, NE Atlantic Ocean. Stockholm Contributions in Geology, 36:1-91.

Black, M. 1971. The systematics of coccoliths in relation to the palaeontological record. In: M. Funnell \& W.R. Riedel (eds.) The Micropaleontology of Oceans, Cambridge University Press, p. 611-624.

Bollmann, J.; Hilbrecht, H. \& Thierstein, H.R. 1993. Evenness and species richness in modern coccolith and foraminifera assemblages. International Nannoplankton Association Newsletter, 15/2:55.

Bown, P.R. 1998. Calcareous nannofossil biostratigraphy. Cambridge, British Micropalaeontological Society Publication Series, Chapman \& Hall, 328 p.

Bown, P.R. \& Newsam, C. 2017. Calcareous nannofossils from the Eocene North Atlantic Ocean (IODP Expedition 342 Sites U1403-1411). Journal of Nannoplankton Research, 37:25-60.

Bown P.R. \& Young J.R. 1998. Techniques. In: P.R. Bown (ed.) Calcareous Nannofossil Biostratigraphy, Kluwer Academic Press, p. 16-28.

Bramlette, M.N. \& Riedel, W.R. 1954. Stratigraphic value of Discoasters and some other microfossils related to recent coccolithophores. Journal of Paleontology, 28:385-403.

Bramlette, M.N. \& Wilcoxon, J.A. 1967. Middle Tertiary calcareous nannoplankton of the Cipero section, Trinidad - W.I. Tulane Studies in Geology and Paleontology, 5:93-131. 
Brand, L.E. 1994. Physiological ecology of marine coccolithophores. In: A. Winter \& W.G. Siesser (eds.) Coccolithophores, Cambridge University Press, p. 39-49.

Brönnimann, P. \& Stradner, H. 1960. Die foraminiferen und Discoasterridenzonen von Kuba und ihre interkontinentale Korrelation. Erdöl Zeitschrift, 76:364-369.

Cachão, M. \& Moita, T. 2000. Coccolithus pelagicus, a productivity proxy related to moderate fronts off Western Iberia. Marine Micropaleontology, 39:131-155. doi:10.1016/S03778398(00)00018-9

Castro, L.N.S.P. 2006. Dinoflagelados e outros palinomorfos do Miocénico do sector distal da Bacia do Baixo Tejo. Universidade Nova de Lisboa, Tese de Doutorado, 380 p.

Cavalier-Smith, T. 1986. The kingdom Chromista: origin and systematics. In: F.E. Round \& D.J. Chapman Progress in Phycological Research, Biopress Ltd. Bristol, p. 309-347.

Chepstow-Lusty, A.; Backman, J. \& Shac-Kleton, N.J. 1989. Comparison of Upper Pliocene Discoaster abundance varitions from North Atlantic Sites 522, 607, 659, 658 and 662: further evidence for marine plankton responding to orbital forcing. Proceedings of the Ocean Drilling Program, Scientific Results, 108:121-141. doi:10.2973/odp.proc.sr.108.122.1989

Chepstow Lusty, A.; Backman, J. \& Shac-Kleton, N.J. 1992. Comparison of Upper Pliocene Discoaster abundance variations from the Atlantic, Pacific and Indian Oceans: the significance of productivity pressure at low latitudes. Memorie di Scienze Geologiche, 44:357-373.

Chira, C. \& Mãrunteanu, M. 1999. Middle Miocene (Lower Badenian) calcareous nannofossils from the Muresholl passageway and Fãget Basin, Romania. Acta Palaeontologica Romaniae, 5:73-82.

Cruces, A.; Lopes, A.; Freitas, M.C. \& Andrade, C. 2002. A Geologia no Litoral. Parte II: Da Lagoa de Albufeira a Setúbal. Lisboa, Agencia Ciência Viva, Faculdade de Ciências da Universidade de Lisboa, $37 \mathrm{p}$.

Deflandre, G. 1952. Classe des Coccolithophoridés. (Coccolithophoridae. Lohmann, 1902). In: P.P. Grassé (ed.) Traité de Zoologie, Masson, p. 439-470.

Dunkley Jones, T.; Bown, P.R. \& Pearson, P.N. 2009. Exceptionally well preserved upper Eocene to lower Oligocene calcareous nannofossils from the Pande Formation (Kilwa Group), Tanzania. Journal of Systematic Palaeontology, 7:359-411. doi:10.1017/S1477201909990010

Edvardsen, B.; Eikrem, W.; Green, J.C.; Andersen, R.A.; Yeo, Moon-Van Der Staay, S. \& Medlin, L.K. 2000. Phylogenetic reconstructions of the Haptophyta inferred from 18S ribosomal DNA sequences and available morphological data. Phycologia, 39:19-35. doi:10.2216/i0031-8884-39-1-19.1

Edwards, A.R. 1968. The calcareous nannoplankton evidence for New Zealand Tertiary marine climate. Tuatara, 16:26-30.

Flores, J.A. \& Sierro, F.J. 1987. Calcareous plankton in the Tortonian/Messinian Transition Series of the northwestern edge of the Guadalquivir Basin. Abhandlungen der Geologischen Bundesanstalt, 39:67-84.

Flores, J.A.; Sierro, F.J. \& Raffi, I. 1995. Evolution of the calcareous nannofossil assemblage as a response to the paleoceanographic changes in the eastern equatorial Pacific Ocean from 4 to 2 Ma. Proceedings of the Ocean Drilling Program, Scientific Results, 138:163-176. doi:10.2973/odp.proc.sr.138.109.1995

Fritsch, F.E. 1929. Evolutionary sequence and affinities among Protophyta. Biological Reviews, 4:103-151. doi:10.1111/j.1469185X.1929.tb00884.x
Gartner, S.; Chen, M.P.; \& Stanton, R.J. 1983. Late Neogene nannofossil biostratigraphy and paleoceanography of the north eastern Gulf of Mexico and adjacent areas. Marine Micropaleontology, 8:17-50. doi:10.1016/0377-8398(83)90003-8

Giraudeau, J. 1992. Distribution of recent nannofossils beneath the Benguela system: southwest African continental margin. Marine Geology, 108:219-237. doi:10.1016/0025-3227(92)90174-G

Haeckel, E. 1894. Systematische Phylogenie der Protisten und Pflanzen. Berlin, Reimer, $420 \mathrm{p}$.

Haq, B.U. 1980. Biogeographic history of Miocene calcareous nannoplankton and paleoceanography of the Atlantic Ocean. Micropaleontology, 26:414-443. doi:10.2307/1485353

Haq, B.U. \& Lohmann, G.P. 1976. Early Cenozoic calcareous nannoplankton biogeography of the Atlantic Ocean. Marine Micropaleontology, 1:119-197. doi:10.1016/03778398(76)90008-6

Hay, W.W. 1977. Calcareous nannofossils. In: A.T.S. Ramsay (ed.) Oceanic Micropalaentology, Academic Press, p. 1055-1200.

Hay, W.W.; Mohler, H.P.; Roth, P.H.; Schmidt, R.R. \& Boudreaux, J.E. 1967. Calcareous nannoplankton zonation of the Cenozoic of the Gulf Coast and Caribbean-Antillean area, and transoceanic correlation. Gulf Coast Association of Geological Societies, Transactions, 17:428-480.

Hay, W.W.; Mohler, H. \& Wade, M.E. 1966. Calcareous nannofossils from Nal'chik (Northwest Caucasus). Eclogae Geologicae Helvetiae, 59:379-399.

Höll, C.; Zonneveld, K.A.F. \& Willems, H. 1998. On the ecology of calcareous dinoflagellates: the Quaternary Eastern Equatorial Atlantic. Marine Micropaleontology, 33:1-25. doi:10.1016/ S0377-8398(97)00033-9

Howe, R.W. \& Sblendorio-Levy, J. 1998. Calcareous nannofossil biostratigraphy and sediment accumulation of turbidite sequences on the Madeira Abyssal Plain, ODP Sites 950-952. Proceedings of the Ocean Drilling Program, Scientific Results, 157:501-520. doi:10.2973/odp.proc.sr.157.147.1998

Jerkovic, L. 1970. Noëlaerhabdus nov. gen. type d'une nouvelle famille de Coccolithophoridés fossiles: Noëlaerhabdaceae du Miocène supérieur de Yugoslavie. Comptes Rendus Hebdomadaires des Séances de l'Académie des Sciences, Série D - Sciences Naturelles, 270:468-470.

Kaenel, E.; Bergen, J.A.; Browning, E.; Blair, S.A. \& Boesiger, T.M. 2017. Uppermost Oligocene to Middle Miocene Discoaster and Catinaster taxonomy and stratigraphy in the circum North Atlantic Basin: Gulf of Mexico and ODP Leg 154. Journal of Nannoplankton Research, 37:215-244

Kaenel, E.P. \& Villa, G. 1996. Oligocene-Miocene calcareous nannofossil biostratigraphy and paleoecology from the Iberia Abyssal Plain. Proceedings of the Ocean Drilling Program, Scientific Results, 149:79-145. doi:10.2973/odp.proc. sr. 149.208 .1996

Kamptner, E. 1927. Beitrag zur Kenntnis adriatischer Coccolithophoriden. Archiv für Protistenkunde, 58:173-184.

Kamptner, E. 1948. Coccolithen aus dem Torton des Inneralpinen Wiener Beckens. Anzeiger. Österreichische Akademie der Wissenschaften, Mathematish-Naturwissenschaftliche, 157:1-16.

Kamptner, E. 1950. Über den submikroskopischen Aufbau der Coccolithen. Anzeiger. Österreichische Akademie der Wissenschaften Mathematische-Naturwissenschaftliche, 87:152-158.

Kamptner, E. 1954. Untersuchungen uber den Feinbau der Coccolithen. Archiv fuer Protistenkunde, 100:1-90. 
Kamptner, E. 1963. Coccolithineen-Skelettreste aus Tiefseeablagerungen des Pazifischen Ozeans. Annalen des Naturhistorischen Museums in Wien, 66:139-204.

Lancis, C.; Tent-Manclús, J.E.; Soria, J.M.; Caracuel, J.E.; Corbí, H.; Dinarès-Turell, J.; Estévez, A. \& Yébenes, A. 2010. Nannoplankton biostratigraphic calibration of the evaporitic events in the Neogene Fortuna Basin (SE Spain). Geobios, 43:201-217. doi:10.1016/j.geobios.2009.09.007

Legoinha, P. 2001. Biostratigrafia de foraminíferos do Miocénico de Portugal. Universidade Nova de Lisboa, Tese de doutorado, $238 \mathrm{p}$.

Lemmermann, E. 1908. Flagellatae, Chlorophyceae, Coccosphaerales und Silicoflagellatae. In: K. Brandt \& C. Apstein (eds.) Nordisches Plankton, Lipsius \& Tischer, p. 1-40.

Lohmann, H. 1902. Die Coccolithophoridae, eine Monographie der Coccolithen bildenden Flagellaten, zugleich ein Beitrat zur Kenntnis des Mittelmeerauftriebs. Archiv fuer Protistenkunde, 1:89-165.

Lohmann, G.P. \& Carlson, J.J. 1981. Oceanographic significance of Pacific late Miocene calcareous nannoplankton. Marine Micropaleontology, 6:553-579. doi:10.1016/03778398(81)90021-9

Manuppella, G.; Antunes, M.T.; Pais, J.; Ramalho, M.M. \& Rey, J. 1999. Notícia explicativa da Folha 38-B (Setúbal) - Carta Geológica de Portugal à escala 1:50.000. Lisboa, Instituto Geológico e Mineiro, Departamento de Geologia, 143 p.

Marsche, I.S. 1978. Contributions à la paléontologie du Miocène moyen continental du Bassin du Tejo. IV Charophytes - Póvoa de Santarém, Pero Fino et Tremês. Ciências da Terra, 4:91-102.

Martini, E. 1971. Standard tertiary and quaternary calcareous nannoplankton zonation. In: A. Farinacci (ed.) Proceedings of the II Planktonic Conference, p. 739-785.

Martini, E. \& Worsley, T. 1971. Tertiary calcareous nannoplankton from the western equatorial Pacific. In: E.L. Winterer et al. Initial Reports of the Deep Sea Drilling Project, Vol. 7, U.S. Government Printing Office, p. 1471-1507. doi:10.2973/dsdp. proc. 7.129 .1971

McIntyre, A. \& Bé, A.W.H. 1967. Modern coccolithophoridae of the Atlantic Ocean: I. Placoliths and cyrtoliths. Deep Sea Research, 14:561-597. doi:10.1016/0011-7471(67)90065-4

Melchiades, F.G. \& Boschi, A.O. 2006. A calcimetria como ferramenta para o controle de bitola em massas de revestimentos cerâmicos fabricados por via seca. Cerâmica Industrial, 11:7-11.

Melinte, M.C. 2005. Oligocene palaeoenvironmental changes in the Romanian Carpathians, revealed by calcareous nannofossils. Studia Geologica Polonica, 124:341-352.

Müller, C. 1970. Nannoplankton aus dem Mittel-Oligozaen von Norddeutschland und Belgien. Neues Jahrbuch für Geologie und Paläontologie, 135:82-101.Müller, C. 1976. Tertiary and Quaternary calcareous nannoplankton in the NorwegianGreenland Sea, DSDP Leg 38. In: M. Talwani et al. Initial Reports of the Deep Sea Drilling Project, Vol. 38, U.S. Government Printing Office, p. 823-841.

Müller, C. 1976, Tertiary and Quaternary calcareous nannoplankton in the Norwegian-Greenland Sea, DSDP, Leg 38. In: Initial Reports of the Deep Sea Drilling Project (Talwani, M., Udintsev, G., White, S.M., Eds.), p. 823-841. doi:10.2973/dsdp. proc.38.126.1976.

Müller, C. 1981. Beschreibung neuer Helicosphaera Arten aus dem Miozan und Revision biostratigraphischer Reichweiten einiger neogener Nannoplankton-Arten. Senckenbergiana Lethaea, 61:427-435.
Muza, J.P.; Wise, S.W. \& Covington, J.M. 1987. Neogene calcareous nannofossils from Deep Sea Drilling Project Site 603, lower continental rise, western North Atlantic: biostratigraphy and correlations with magnetic and seismic stratigraphy. In: J.E. van Hinte et al. Initial Reports of the Deep Sea Drilling Project, Vol. 93, U.S. Government Printing Office, p. 593-616. doi:10.2973/ dsdp.proc.93.115.1987

Nascimento, A. 1983. The Ostracoda fauna of the Portuguese Neogene and its relationhips to those from the Atlantic and Mediterranean Basins. In: R.F. Maddocks (ed.) Applications of Ostracoda, Proceedings of the Eighth International Symposium on Ostracoda, University of Houston-University Park, p. 429-436.

Okada, H. \& Bukry, D. 1980. Supplementary modification and introduction of code numbers to the low-latitude coccolith biostratigraphic zonation (Bukry, 1973; 1975). Marine Micropaleontology, 5:321-325. doi:10.1016/03778398(80)90016-X

Okada, H. \& McIntyre, A. 1979. Seasonal distribution of modern coccolithophores in the westen North Atlantic Ocean. Marine Biology, 54:319-328. doi:10.1007/BF00395438

Pais, J.; Cunha, P.; Legoinha, P.; Dias, R.; Pereira, D. \& Ramos, A. 2013. Cenozoico das Bacias do Douro (sector ocidental), Mondego, Baixo Tejo e Alvalade. In: R. Dias, A. Araújo, P. Terrinha \& J.C. Kullberg (eds.) Geologia de Portugal - Vol. II Geologia Meso-cenozoica de Portugal, Escolar, p. 461-532.

Perch-Nielsen, K. 1985. Cenozoic calcareous nannofossils. In: H.M. Bolli, J.B. Saunders \& K. Perch-Nielsen (eds.) Plankton stratigraphy, Cambridge University Press, p. 427-554.

Poche, F. 1913. Das System der Protozoa. Archiv für Protistenkunde, 30:125-321.

Raffi, I.; Backman, J.; Fornaciari, E.; Palike, H.; Rio, D.; Lourens, L. \& Hilgen, F. 2006. A review of calcareous nannofossil astrobiochronology encompassing the past 25 million years. Quaternary Science Reviews, 25:3113-3137. doi:10.1016/j. quascirev.2006.07.007

Rahman, A. \& Roth, P.H. 1990. Late Neogene paleoceanography and paleoclimatology of the Gulf of Aden region based on calcareous nannofossils. Paleoceanography, 5:91-107. doi:10.1029/ PA005i001p00091

Rai, J. \& Singh, A. 2010. Calcareous nannofossils and their applications. Gondwana Geological Magazine, 25:49-159.

Raven, P.H.; Evert, R.F. \& Eichhorn, S.E. 2007. Biologia Vegetal. $7^{\text {a }}$ ed. Rio de Janeiro, Editora Guanabara Koogan, 830 p.

Roth, P.H. 1970. Oligocene calcareous nannoplankton biostratigraphy. Eclogae geologicae Helvetiae, 63:799-881.

Roth, P.H. 1994. Distribution of coccoliths in ocean sediments. In: A. Winter \& W.G. Siesser (eds.) Coccolithophores, Cambridge University Press, p. 199-218.

Rothmaler, W. 1951. Die Abteilungen und Klassen der Pflanzen. Feddes Reportorium, 54:256-266.

Sanders, H.L. 1969. Benthic marine diversity and the stability time hypothesis. In: G.M. Woodwell \& H.H. Smith (eds.) Diversity and Stability in Ecological Systems, Brookhaven National Laboratory, p. 71-81.

Sblendorio-Levy, J. \& Howe, R.W. 1998. Calcareous nannofossil biostratigraphy of site 953, Canary Basin, northeastern North Atlantic. In: P.P.E. Weaver et al. Proceedings of the Ocean Drilling Program, Scientific Results, Vol. 157, p. 83-96. doi:10.2973/odp.proc.sr.157.107.1998 
Schiller, J. 1930. Coccolithineae. In: L. Rabenhorst (ed.) Kryptogamen-Flora von Deutschland, Österreich und der Schweiz, Akademische Verlagsgesellschaft, p. 89-267.

Schmidt, R.R. 1978. Calcareous nannoplankton from the western North Atlantic, DSDP Leg 44. In: W.E. Benson et al. Initial Reports of the Deep Sea Drilling Project, Vol. 44, p. 703-729. doi:10.2973/dsdp.proc. 44.133 .1978

Schwarz, E.H.L. 1894. Coccoliths. Annals and Magazine of Natural History, 14:341-346.

Scotese, C. 2014. Atlas of Neogene Paleogeographic Maps (Mollweide Projection), Maps 1-7, Vol. 1 - The Cenozoic, PALEOMAP Atlas for ArcGIS, PALEOMAP Project, Evanston, IL.

Shannon, C.E. \& Weaver, W. 1949. The Mathematical Theory of Communication. University of Illinois Press, $125 \mathrm{p}$.

Steinmetz, J.C. 1979. Calcareous nannofossils from the North Atlantic Ocean, Leg 49. In: B.P. Luyendyk et al. Initial Reports of the Deep Sea Drilling Project, Vol. 49, U.S. Government Printing Office, p. 519-531. doi:10.2973/dsdp.proc.49.116.1979

Takayama, T. 1967. First report on nannoplankton of the Upper Tertiary and Quaternary of the southern Kwanto region, Japan. Jahrbuch der Geologischen Bundesanstalt, 110:169-198.

Tan, S.H. 1927. Discoasteridae incertae sedis. Proceedings of the Koninklijke Nederlandse Akademie van Wetenschappen, Section of Science, 30:411-419.

Tangen, K.; Brand, L.E.; Blackwelder, P.L. \& Guillard, R.R.L. 1982. Thoracosphaera heimii (Lohmann) Kamptner is a dinophtye: obsevations on its morphology and life cycle. Marine Micropaleontology, 7:193-212.
Varol, O. 1998. Paleogene. In: P.R. Bown (ed.) Calcareous Nannofossils Biostratigraphy, British Micropaleontological Society Publications Series, Chapman \& Hall, p. 201-224.

Wade, B.S. \& Bown, P.R. 2006. Calcareous nannofossils in extreme environments: the Messinian Salinity Crisis, Polemi Basin, Cyprus. Palaeogeography, Palaeoclimatology, Palaeoecology, 233:271-286. doi:10.1016/j.palaeo.2005.10.007

Wallich, G.C. 1877. Observations on the Coccosphere. Annals and Magazine of Natural History, 4:342-350.

Young, J.R. 1998. Neogene nannofossils. In: P.R. Bown (ed.) Calcareous Nannofossil Biostratigraphy, Kluwer Academic Publications, p. 225-265.

Young, J.R. \& Bown, P.R. 1997. Cenozoic calcareous nannoplankton classification. - Proposals for a revised classification system for calcareous nannoplankton. Journal of Nannoplankton Research, 19:15-47.

Young, J.R.; Bown P.R. \& Lees J.A. 2018. Nannotax website3. International Nannoplankton Association. Accessed 22 Feb. 2020 URL: http://www.mikrotax.org/Nannotax3

Young, J.R.; Geisen, M.; Cros, L.; Kleijne, A.; Probert, I.; Sprengel, C. \& Ostergaard, J.B. 2003. A guide to extant coccolithophore taxonomy. Journal of Nannoplankton Research, 1:1-125.

Young, J.R.; Jordan, R.W. \& Cros, L. 1998. Notes on nannoplankton systematics and life-cycles - Ceratolithus cristatus, Neosphaera coccolithomorpha and Umbilicosphaera sibogae. Journal of Nannoplankton Research, 20:89-99.

Received in 18 February, 2020; accepted in 28 September, 2020. 